JGG 2021;69:208-210

doi: $10.36150 / 2499-6564-N 262$

\title{
Management of balance problems in an elderly with multiple system atrophy with predominant cerebellar ataxia (MSA-C) and sick sinus syndrome
}

\author{
Amber Eker ${ }^{1}$, Pembe Hare Yigitoglu², Hamza Duygu ${ }^{3}$, Ersin Tan ${ }^{4}$ \\ ${ }^{1}$ Eastern Mediterrenean University Faculty of Medicine, Famagusta, Northern Cyprus, Mersin \\ 10 Turkey; ${ }^{2}$ Near East University Faculty of Medicine Department of Physical Medicine and \\ Rehabilitation, Nicosia, Northern Cyprus; ${ }^{3}$ Near East University Faculty of Medicine Department of \\ Cardiology, Nicosia, Northern Cyprus; ${ }^{4}$ Hacettepe University Faculty of Medicine Department of \\ Neurology, Ankara, Turkey
}

Received: September 04, 2020

Accepted: March 29, 2021

\section{Correspondence}

Amber Eker

Eastern Mediterrenean University Faculty of Medicine, Famagusta, Northern Cyprus, Mersin 10 Turkey. E-mail: amber.eker@emu.edu.tr

How to cite this article: Eker A, Yigitoglu $\mathrm{PH}$, Duygu $\mathrm{H}$, et al. Management of balance problems in an elderly with multiple system atrophy with predominant cerebellar ataxia (MSA-C) and sick sinus syndrome. Journal of Gerontology and Geriatrics 2021;69:208-210. https://doi. org/10.36150/2499-6564-N262

(C) Copyright by Società Italiana

di Gerontologia e Geriatria (SIGG)

\section{cc) (i) (9)}

\section{OPEN ACCESS}

This is an open access article distributed in accordance with the CC-BY-NC-ND (Creative Commons Attribution-NonCommercial-NoDerivatives 4.0 International) license. The article can be used by giving appropriate credit and mentioning the license, but only for non-commercial purposes and only in the original version. For further information: https://creativecommons.org/licenses/by-nc-nd/4.0/deed.en
Multiple system atrophy (MSA) is a late-onset, sporadic, progressive, neurodegenerative disorder clinically characterized by autonomic failure and either poorly levodopa-responsive parkinsonism or cerebellar ataxia. There isn't any effective treatment on disease progression. Current therapeutic strategies are primarily based on dopamine replacement and improvement of autonomic failure. Clonazepam, propranolol, baclofen, amantadine, gabapentin, buspirone have also been used for symptomatic treatment of ataxia in MSA patients, but their efficacy known modest and transient. Herein we describe a MSA-C patient with disabling ataxia and sick sinus syndrome who showed distinct improvement in her symptoms after amantadine treatment and cardiac pacemaker implantation.

Key words: multiple system atrophy, spinocerebellar degeneration, ataxia, amantadine, sick sinus syndrome, gait ataxia

\section{INTRODUCTION}

Multiple system atrophy (MSA) is a late-onset, sporadic, progressive, neurodegenerative disorder clinically characterized by autonomic failure and either poorly levodopa-responsive parkinsonism or cerebellar ataxia. Neuroimaging supporters are; atrophy on magnetic resonance imaging (MRI) of putamen, middle cerebellar peduncule and pons or hypometabolism on18 fluoro-2-deoxyglucose positron emission tomography (PET) in putamen or presynaptic nigrostriatal dopaminergic denervation on single photon emission computed tomography SPECT or PET. There are two clinical subtypes of MSA distinguished by the predominant motor features: the parkinsonian variant (MSA-P) and the cerebellar variant (MSA-C). Neuropathologically it is defined by alpha-synuclein positive glial cytoplasmic inclusions and striatonigral and/or olivopontocerebellar neurodegeneration ${ }^{1,2}$. MSA is a rare neurodegenerative disorder with no effective treatment on disease progression. Current therapeutic strategies are primarily based on dopamine replacement and improvement of autonomic failure ${ }^{3}$. Herein we describe a MSA-C patient with disabling balance problems who showed distinct improvement after amantadine treatment and cardiac pacemaker implantation. 


\section{CASE REPORT}

74 years old woman referred to our clinic with balance problems in her walking which last for one year and progress during last one month after a diarrhea attack. She uses levothyroxine for her hypothyroidism and metoprolol for her long term rhythm problems. Her neurologic examination revealed ataxic gate and difficulty in tandem walk, also deep tendon reflexes bilaterally increased in both upper and lower extremities. There weren't any oculomotor abnormality, dysarthria, pathologic reflexes and extrapyramidal signs. We didn't observe any decrease in her blood pressure when getting upright position. Her cranial MRI showed T2-weighted hyperintense horizontal band in pons (Fig. 1a) and putaminal rim (Fig. 1b). Somatosensory evoked potentials were normal and help us to exclude a sensory pathway related balance problem. Heart rate variability test could not be performed because of her frequent bradycardia attacks and arrhythmia. Her holter ECG evaluation findings were resemble with sick sinus syndrome. Her detailed neurocognitive evaluation revealed mild executive dysfunction without any memory problem.

To demonstrate her severity of ataxia, we measured her walking capacity, gait speed, standing capacities in different positions and body sway with International Cooperative Ataxia Rating Scale (ICARS) before and after the treatment of amantadine. She could walk without support but with considerable staggering and difficulties in half turn and her gait speed was slightly reduced. She couldn't be able to stand in tandem position. We observed severe body sway especially when eyes were closed. She also had slight trunk ataxia. Total ICARS score had been calculated 14 in her first visit. After the usage of Amantadine 200 mg/day for 1 month, she subjectively described great relief. Additionally her neurologic evaluation revealed that she was able to walk with feet in tandem position and her gait speed was

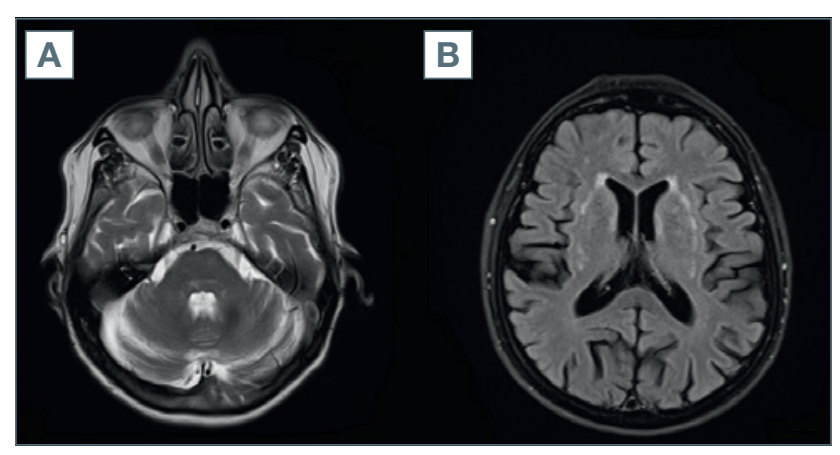

Figure 1. A) horizontal T2 hyperintense band in pons on T2weighted images; B) T2 hyperintense putaminal rim on fluid attenuation inversion recovery (FLAIR) images. marked increased. She could stand feet together and she had only slight body oscillations in standing and sitting positions. ICARS score was calculated as 4 . Besides the recovering ataxic symptoms she had continuing light headedness attacks. Because of the diagnosis of sick sinus syndrome with frequent blocks and bradycardia attacks, cardiology department implanted dual chamber cardiac pacemaker (DDD-R, MR compatible). She described total recovery in light headedness attacks after the operation.

\section{DISCUSSION}

Carefully evaluation of the adult patients with sporadic, progressive ataxia is really important. Metabolic, paraneoplastic, infectious, autoimmune, hereditary conditions and degenerative disorders must be considered in the differential diagnosis of these patients. We differentiated other hereditary disorders and acquired etiologies including metabolic deficiencies, infections, autoimmune conditions and tumors from MSA-C in this patient by the results of detailed disease-course history, family history, neurological examination, brain magnetic resonance imaging, biochemical and other radiological investigations.

As the presence of both motor and autonomic symptoms is essential for the current diagnostic criteria, early diagnosis is difficult when patients present with isolated autonomic failure or motor symptoms in early stages. MSA can show isolated autonomic failure prior to reaching the full-blown stage ${ }^{2}$. Furthermore, ataxia of gait is the most common disabling motor feature of MSA-C initially. This disease progresses more rapidly than other late-onset sporadic ataxias; typically a patient becomes wheelchair dependent by 5 years after onset and mean survival is about 9-10 years after symptom onset 2,3 .

Our patient has bradycardia attacks for many years without any other cardiovascular problem. After the explosion of ataxia that cause severe disability in her daily life she referred to our department. Her evaluation revealed early autonomic disturbances, then additional disabling cerebellar ataxia, pyramidal signs and subcortical cognitive deficit.

Cognitive impairment may also accompany to the primary features in MSA. Researches show that subcortical type cognitive decline observe in MSA-C ${ }^{2}$. Our patients detailed neurocognitive test result also showed us a subcortical mild deficit with problem in executive functions.

Abnormalities that may observe in MSA on conventional MRI include atrophy of the putamen, pons, middle cerebellar peduncles, and cerebellum. Two specific findings often cited as hallmarks for MSA are, slit-like, 
marginal hyperintensity in the putamen predominantly in posterolateral portion (putaminal hyperintense rim) and the hot-cross bun sign, consisting of a cruciform pattern of hyperintensity in the basis pontis visible on T2-weighted and proton density-weighted MRI. Widespread neurodegeneration in MSA give explanation of principal and additional features of the disease ${ }^{2}$. In addition to her clinical symptoms her typical MRI findings are also resemble with probable MCA-C in this patient. Clonazepam, propranolol, baclofen, amantadine, gabapentin, buspirone have been used for symptomatic treatment of ataxia in MSA patients. But the researches emphasize the modest and transient efficacy in retrospective data analysis ${ }^{3}$. There are also some clinical trials on the positive effect of the amantadine treatment on ataxia in different type of degenerative ataxias ${ }^{4}$. Amantadine was originally introduced as an antiviral agent but it has been shown to antagonize $\mathrm{N}$ methyl-D-aspartate (NMDA) receptors and stimulate dopamine release, The rationale of amantadine use in symptomatic management of ataxias can be explained by its dopamine replacement effect and by direct involvement of NMDA in glutamate mediated neurotoxicity in cerebellar granular cells ${ }^{4}$.

She also had bradycardia attacks that cause light headedness additional to her cerebellar dysfunction related balance problems. The common finding is orthostatic hypotension in the autonomic involvement in this kind of neurodegenerative disorders but also hypervagotonia and related rythm problems may observe and cause additional disability on balance and more devastatingly life threatening conditions. Sick sinus syndrome only report in few papers and in the patients who have parkinsonian features ${ }^{5}$. Sick sinus syndrome risk increase by ageing and less probably it may observe incidentally in our old patient. But we never underdiagnosed this syndrome besides the common orthostatic hypotension because this hypervagotonic conditions may need special treatment approach. Our patient who has not any orthostatic hypotension describe total recovery in light headedness and near syncope attacks after cardiac pacemaker implantation. Additionally she can start physiotherapy which will continue in her later life. Physiotherapy remains the best therapeutic option also accept as a first line treatment for cerebellar ataxia in MSA also in dizziness after all secondary reasons elimination ${ }^{3,6}$

To conclude, amantadine seems a safe and effective symptomatic treatment on cerebellar dysfunctions especially in the mild forms of MSA-C. Double blind placebo control trials are needed to establish this effect in larger groups who have MSA-C or other degenerative ataxias. Lastly, fighting with the cerebellar and autonomic system related dysfunction have great effect on the patients daily life in MSA. Even if it is a progressive disease it is important to bring the patient a comfortable life period with all recent opportunities without thinking that the relief will be temporary.

Ethical consideration

Patient consent was obtained.

\section{Acknowledgement}

None.

\section{Funding}

None.

\section{Conflict of interest}

The researchers claim no conflicts of interest.

\section{Author contributions}

Conceptualization,AE,PHY;Data

curation,AE,PHY,HD,ET; Methodology,AE,PHY,HD;

Project administration, AE, PHY; Resources,

AE,PHY,HD,ET;Supervision, AE, ET; Writing - original

draft AE,PHY; Writing - review \& editing HD,ET.

\section{References}

1 Gilman S, Wenning GK, Low PA, et al. Second consensus statement on the diagnosis of multiple system atrophy. Neurology 2008;71:670-676. https://doi.org/10.1212/01. wnl.0000324625.00404.15

2 Watanabe H, Riku Y, Hara K, et al. Clinical and imaging features of multiple system atrophy: challenges for an early and clinically definitive diagnosis. J Mov Disord 2018;11:107120. https://doi.org/10.14802/jmd.18020

3 Perez-Lloret S, Flabeau O, Fernagut PO, et al. Current concepts in the treatment of multiple system atrophy. Mov Disord Clin Pract 2015;2:6-16. https://doi.org/10.1002/ mdc3.12145

4 Botez Ml, Botez-Marquard T, Elie R, et al. Amantadine hydrochloride treatment in heredodegenerative ataxias: a double blind study. J Neurol Neurosurg Psych 1996;61:259-264. https://doi.org/10.1136/jnnp.61.3.259

5 Adamec I, Klepac N, Milivojević I, et al. Sick sinus syndrome and orthostatic hypotension in Parkinson's disease. Acta Neurologica Belgica 2012;112:295-297. https://doi. org/10.1007/s13760-012-0034-0

6 Rhee CK, Kim JS, Chung YW, et al. Clinical features and management of Dizziness in the aged. Ann Geriatr Med Res 2005;9:91-97. 